\title{
CORRECTION
}

\section{Correction to: Comment on: 'Paracentral acute middle maculopathy and acute macular neuroretinopathy following SARS-CoV-2 infection'}

Asterios Diafas (iD, Nima Ghadiri (iD, Nick Beare (D), Savita Madhusudhan, lan Pearce and Shi Zhuan Tan

(c) The Author(s), under exclusive licence to The Royal College of Ophthalmologists 2021

Eye (2022) 36:1522; https://doi.org/10.1038/s41433-021-01752-2

Correction to: Eye https://doi.org/10.1038/s41433-021-01709-5, published online 02 August 2021
Unfortunately, the word "neuroretinopathy" was written incorrect in the title.

The original article has been corrected. 